\title{
NONLINEAR EXTENSIONS OF A FRACTAL-MULTIFRACTAL APPROACH FOR ENVIRONMENTAL MODELING
}

\author{
Andrea Cortis \\ Earth Sciences Division \\ Lawrence Berkeley National Laboratory, Berkeley, CA 94720 \\ acortis@lbl.gov \\ Carlos E. Puente \\ Department of Land, Air and Water Resources \\ University of California, Davis, CA 95616 \\ cepuente@ucdavis.edu \\ Bellie Sivakumar \\ Department of Land, Air and Water Resources \\ University of California, Davis, CA 95616 \\ sbellie@ucdavis.edu
}

\begin{abstract}
We present the extension of a deterministic fractal geometric procedure aimed at representing the complexity of the spatio-temporal patterns encountered in environmental applications. The original procedure, which is based on transformations of multifractal distributions via fractal functions, is extended through the introduction of nonlinear perturbations to the underlying iterated linear maps. We demonstrate how the nonlinear perturbations generate yet a richer collection of patterns by means of various simulations that include evolutions of patterns based on changes in their parameters and in their statistical and multifractal properties. It is shown that the nonlinear extensions yield structures that closely resemble complex hydrologic temporal data sets, such as rainfall and runoff time series, and width-functions of river networks as a function of distance from the basin outlet. The implications of this nonlinear approach for environmental modeling and prediction are discussed.
\end{abstract}




\section{Introduction}

Mathematical methods based on fractal geometry and chaos theory have now long been used as the language for the description of the complexities so often encountered in natural phenomena (e.g., Mandelbrot, 1983; Lorenz, 1993). These notions, however, are oftentimes insufficient to study, on an individual basis, the incredible variety of the patterns observed in nature. Given that natural sets (i.e., time series, spatial patterns, space-time sets) are typically erratic, noisy, intermittent, non-smooth, or in short "random," it has become natural to model them using stochastic (fractal) theories. Stochastic fractal modeling has resulted in a variety of approaches that, while preserving some of the relevant statistical and physical attributes of the records (e.g., autocorrelation function, power spectrum, moments, multifractal spectrum, etc.), are typically unable to capture the specific details and textures present in individual sets.

As stochastic approaches, by definition, can only generate plausible realizations preserving only some features of the data, and as studies of nonlinear dynamics have revealed that details indeed matter (e.g., in climate studies; Lorenz 1993), the following questions become inevitable: (1) Is it possible to devise a modeling approach that faithfully models individual patterns capturing not only the overall trends and statistical features of the records but also their inherent details? (2) Can such an approach be defined without resorting to the concept of randomness, implying an inherent hidden order in complexity as in deterministic chaos? (3) Can such an approach, by the capturing of details, be helpful in studying the underlying dynamics of such sets?

It is now well known that a large class of deterministic fractal sets can be generated via iterations of simple linear maps (e.g., Barnsley, 1988). Puente (1992) described how fractal interpolation functions (defined as the attractors of simple linear maps) transform multifractal distributions into deterministic derived measures that mimic the complexity of patterns found in nature. The present work reviews and extends this deterministic fractal geometric approach of Puente (1992). The extensions entail adding bounded nonlinear perturbations to the above iterated linear maps to produce yet more exotic attractors and subsequent deterministic derived measures. As shall 
be demonstrated herein, the nonlinear perturbations uncover a vast class of relevant patterns over one dimension that closely resemble those found in a variety of environmental applications. These patterns are parsimoniously encoded via only a few geometric parameters (i.e., the quantities that define the involved maps and perturbations and also the multifractal distribution).

The organization of this paper is as follows. Given first is a review of the original mathematical construction and a description of the nonlinear extensions. This is followed by a variety of interesting examples that include a host of evolutions varying a few parameters and that contain a statistical and multifractal analysis of the patterns. The relevance of this approach to environmental modeling is discussed and directions for future work are sketched.

\section{The Fractal Multifractal Approach}

The graph $G$ of a fractal interpolating function, from $x$ to $y$ and passing by $N+1$ points on the plane $\left\{\left(x_{n}, y_{n}\right) ; x_{0}<\ldots<x_{N}\right\}$, is defined by iterating $N$ linear maps, $n=1, \ldots, N$ (e.g., Barnsley, 1986):

$$
w_{n}\left(\begin{array}{l}
x \\
y
\end{array}\right)=\left(\begin{array}{cc}
a_{n} & 0 \\
c_{n} & d_{n}
\end{array}\right)\left(\begin{array}{l}
x \\
y
\end{array}\right)+\left(\begin{array}{c}
e_{n} \\
f_{n}
\end{array}\right),
$$

such that $\left|d_{n}\right|<1$ and

$$
w_{n}\left(\begin{array}{l}
x_{0} \\
y_{0}
\end{array}\right)=\left(\begin{array}{l}
x_{n-1} \\
y_{n-1}
\end{array}\right), w_{n}\left(\begin{array}{l}
x_{N} \\
y_{N}
\end{array}\right)=\left(\begin{array}{l}
x_{n} \\
y_{n}
\end{array}\right) .
$$

The initial conditions in equations (2) lead to simultaneous sets of linear equations that allow calculation of the parameters $a_{n}, c_{n}, e_{n}$, and $f_{n}$ in terms of the interpolating points and the vertical scalings parameters, $d_{n}$ :

$$
\begin{aligned}
a_{n} & =\frac{x_{n}-x_{n-1}}{x_{N}-x_{0}} \\
c_{n} & =\frac{y_{n}-y_{n-1}-d_{n}\left(y_{N}-y_{0}\right)}{x_{N}-x_{0}} \\
e_{n} & =x_{n-1}-x_{0} \cdot a_{n} \\
f_{n} & =y_{n-1}-c_{n} \cdot x_{0}-d_{n} \cdot y_{0}
\end{aligned}
$$


Equations (2) ensure that $G$ (the attractor obtained by arbitrarily iterating the linear maps) exists and that it is a continuous function that contains the initial interpolating points (e.g., Barnsley, 1986). As the same attractor $G$ is found irrespective of how the iterations are performed (i.e., with fair or biased proportions on the maps $w_{n}$ ), such a unique set is fully deterministic. Depending on the parameters, $G$ turns out to have a fractal dimension $D \in$ $[1,2)$ (Barnsley, 1988).

As the process of iterations is carried out, effectively sampling $G$ point by point, not only is the attractor being computed but also a unique invariant measure over $G$ is induced, which reflects how the attractor is being filled up. The existence of such a measure allows computing unique (and once again fully deterministic) projections over the coordinates $x$ and $y$ (say $d x$ and $d y$ ) that turn out to have interesting shapes, as found in a variety of environmental applications and beyond (e.g., Puente, 1996, 2004).

Figure 1 shows an example of these ideas for a fractal interpolating function (having fractal dimension $D=1.485)$ that passes by $\{(0,0),(1 / 2,-0.35),(1,-$ $0.2)\}$ and is based on the scalings $d_{1}=-0.8$ and $d_{2}=-0.6$. In addition to the graph of the attractor $G$, the figure also includes the implied projections $d x$ and $d y$ of the unique measure found over $G$ (having $2^{12}=4096$ bins) when the corresponding mappings $w_{1}$ and $w_{2}$ are iterated ( $2^{19}$ times) according to a $30-70 \%$ proportion, using independent pseudo-random numbers, starting the process from the mid-point $(1 / 2,-0.35)$.

As the $x$ coordinate of the linear maps does not depend on $y$ (as implied by the zero value in the first component of Equation (1)), the measure $d x$ is a deterministic binomial multifractal with parameter $p=0.3$ as defined via a multiplicative cascade (Mandelbrot, 1989). The measure $d y$, being related to $d x$ via the fractal interpolating function, turns out to be the derived measure of $d x$ via the function that $G$ graphs and is, hence, computed adding all measure values over $x$ that correspond to a given value of $y$ (e.g., Puente, 1994, 1996). Notice that this projection may be computed at a general angle $\theta$, other than zero degrees as shown in Figure 1.

As may be seen, the procedure leads to complex and "random-looking" measures $d y$ (i.e., the projection) that resemble environmental time series, such as a rainfall data set as a function of time (Puente and Obregón, 1996). As multifractal measures are relevant in turbulence studies (e.g., Meneveau 
and Sreenivasan, 1987), the projection sets, $d y$, may be assigned an interpretation as reflections or transformations of turbulence (Puente and Sivakumar, 2007).

\section{Nonlinear Extensions of the Fractal Multifractal Approach}

A suitable way to extend the fractal-multifractal approach is to add a bounded nonlinear perturbation $g(y)$ on the $y$ component of the linear maps, as follows:

$$
w_{n}\left(\begin{array}{l}
x \\
y
\end{array}\right)=\left(\begin{array}{cc}
a_{n} & 0 \\
c_{n} & d_{n}
\end{array}\right)\left(\begin{array}{c}
x \\
y+g(y)
\end{array}\right)+\left(\begin{array}{c}
e_{n} \\
f_{n}
\end{array}\right) .
$$

This yields, using the same initial conditions as before (equations (2)), the following modified parameters $c_{n}$ and $f_{n}$ that now depend on the specific choice of the nonlinear function $g$ and the scaling parameters $d_{n}$ :

$$
\begin{aligned}
& c_{n}=\frac{y_{n}-y_{n-1}-d_{n}\left(y_{N}+g\left(y_{N}\right)-y_{0}-g\left(y_{0}\right)\right)}{x_{N}-x_{0}} \\
& f_{n}=y_{n-1}-c_{n} \cdot x_{0}-d_{n} \cdot\left(y_{0}+g\left(y_{0}\right)\right)
\end{aligned}
$$

As an example, Figure 2 shows what is obtained (for the same set of parameters used for the linear case in Figure 1) while adding a cosine perturbation of the form $g(y)=A \cdot \cos (\omega \cdot y)$, for an amplitude $A=0.5$ and a frequency $\omega=1$. As may be noticed, unlike the linear case in which successive points agglomerate into a single continuous function, this nonlinear case results in an attractor that is a sparse collection of points. Such a set, obviously not a continuous function, is found to repeat itself under increasing magnification and has a similar fractal dimension as the interpolating function in Figure 1 (i.e., $D \approx 1.485$ ). At the end, however, notice how the same multifractal measure $d x$ gives rise now to other interesting projection sets $d y$, whose overall features and textures are similar to those observed in Figure 1.

As a way of comparison, Figure 3 shows a statistical-multifractal analysis of the "signals" in Figures 1 and 2. In addition to the projections themselves (plotted left to right rather than bottom to top), the figures also include the records' autocorrelation functions (with the $\pm e^{-1}$ levels highlighted), their power spectra (plotted in a log-log scale) and their multifractal spectra (i.e. the " $f$ vs. $\alpha$ " curve, Puente and Obregón, 1999). 
Even if the locations of the main peaks vary, both data sets share a similar degree of complexity, as indicated by similar decay in autocorrelation functions, power-law scaling in their power spectra $\left(S(\omega) \sim \omega^{\beta}\right)$, and multifractal properties (as reflected by the close inverted parabolas in their multifractal spectra). More specifically, one finds that both signals share similar statistical-multifractal qualifiers, as follows. They have, for the linear and nonlinear cases, respectively, (a) fractal dimensions for the signals $D_{d y}$ of 1.43 and 1.37 (not the dimension of their defining attractors $G$ ), (b) correlation scales $\tau_{e}$ (defined when correlation reaches $e^{-1}$ ) of 101 and 80 lags, (c) spectral exponents $\beta$ of -1.21 and -1.08 , and (d) entropy dimensions $D_{1}$ (the shown intersection between the multifractal spectrum and the $f=\alpha$ line) of 0.88 and 0.85 .

Overall, these characteristics (for both the linear and the nonlinear cases) are similar to those found in practical environmental applications, such as in rainfall and runoff studies. As such, the enhanced fractal-multifractal framework provided by the addition of nonlinear perturbations becomes a viable alternative worthy of further investigation. An initial exploration of the vast array of (deterministic) sets that may be generated via this nonlinear setting is presented next.

\section{Sample Patterns via the Nonlinear Fractal-Multifractal Approach}

Figure 4 presents a sensitivity analysis around the pattern generated via the linear fractal-multifractal approach shown in Figure 1 (now portrayed on the top of Figure 4), by adding the aforementioned cosine perturbation, varying the amplitude $A$ from 0 (i.e., the linear case) to 0.5 in increments of 0.125 , while having the frequency parameter fixed at $\omega=2$. As may be observed, the main chunks as well as the major peaks of these interesting sets, all of which may very well represent reasonable variations of a given phenomena (e.g., rainfall), travel to the right as $A$ is increased, while leaving close-to-zero values, on the right hand side of the domain, mostly unchanged. As reported in the caption, although these patterns share a similar fractal dimension $D_{d y}$ close to the average value of 1.30, they do not exhibit a monotonic behavior in their other main statistical-multifractal qualifiers. For instance, there are up and down variations in: (a) the fractal dimension of their underlying 
attractors $D$, (b) their correlation scales $\tau_{e},(\mathrm{c})$ the negative of the spectral exponents $\beta$, and (d) their entropy dimensions $D_{1}$ (even if only slightly for the first four sets).

It is worth noticing that the last set, corresponding to the lowest attractor dimension $D$, exhibits more areas of inactivity as compared to the other sets. This happens because such an attractor is less capable than the others of filtering the intermittencies and overall complexity of the parent multifractal measure $d x$. Due to this fact, the autocorrelation function for such a case decays very fast to zero, the spectral exponent yields a case close to " $1 / f$ " noise, and the entropy dimension is low, indicating a high degree of disorganization.

Figure 5 shows a similar sensitivity analysis to the one just mentioned, but varying the frequency parameter $\omega$ on the cosine perturbation, while leaving the amplitude fixed at $A=0.5$. The sets shown correspond to equal increments of $\omega$ values ranging from 0.5 (top) to 2.5 (bottom), and, hence, the second pattern from the top corresponds to the one already presented in Figure 2. Contrary to the previous sensitivity analysis, the signals here maintain the locations of their main chunks and major peaks when the frequency parameter is varied. Also, some noticeable trends may be discerned regarding their statistical-multifractal attributes, as follows: (a) the fractal dimensions of the attractors $D$ and signals $D_{d y}$ decrease, (b) their correlation scales $\tau_{e}$ tend to decrease, and (c) the entropy dimensions $D_{1}$ also decrease.

Although results regarding the power spectrum exponent $\beta$ are not equally conclusive, what was said of the last pattern in Figure 4 may also be said of the last two sets in Figure 5. As the fractal dimensions of their underlying attractors $D$ are significantly lower than the other signals, such attractors reflect, to a larger degree than those with higher dimensions, the intricacies of the highly irregular parent multifractal measure $d x$. Overall and for the particular set of parameters used, an increasingly oscillating perturbation (i.e., as $\omega$ increases) has the ultimate effect of smoothing, by its increasing ups and downs, an underlying fractal attractor $G$, and if a multifractal appears in $x$ such shall result in derived sets with increasing complexity (i.e., lower $\tau_{e}$ and $\left.D_{1}\right)$. However, care must be exercised, as shall be noted later, for an increase in frequency does not necessarily imply a smoothing of an attractor.

As a means of continuing the exploration, Figure 6 presents other inter- 
esting patterns obtained via a sensitivity analysis of another more erratic set as computed via the linear fractal-multifractal methodology (Puente, 2004, Figure 7). Specifically, all patterns correspond to the interpolating points $\{(0,0),(0.3,1),(1,0)\}$, vertical scalings $d_{1}=0.4, d_{2}=-0.5$, a parent multifractal parameter $p=0.4$, a projection angle $\theta=-20$ degrees, when varying the cosine amplitude $A$ from 0 (i.e., the linear case) to 1 in increments of 0.25 , while having the frequency set at $\omega=0.05$. Given that the fractal dimensions of the attractors are lower than 1.2, all patterns exhibit an intense level of activity reminiscent of "cyclical" environmental signals. As already found in Figure 4, when the amplitude increases significantly, the resulting signal contains periods of no activity and has an increasing complexity.

Notice how these "simulations," although fully deterministic, may be used to fully describe the apparent randomness found in applications. This is indeed a remarkable feature of these ideas that cannot be matched by stochastic approaches, which by definition can only get realizations that match some of the statistical-multifractal properties of the records. Although these patterns show extremely similar statistical-multifractal characteristics, these sets are indeed quite different from one another, strongly reminding us that statisticalmultifractal qualifiers do not fully characterize the variety of patterns that can be generated through the (nonlinear) fractal-multifractal procedure, as it is most likely found with natural sets.

Figure 7 further explores the parameter space of the nonlinear projections via a sensitivity analysis on the frequency $\omega$, as in Figure 5. Specifically, the patterns correspond to the interpolating points $\{(0,0),(1 / 2,5),(1,4)\}$, vertical scalings $d_{1}=0.9, d_{2}=-0.4$, a parent multifractal parameter of $p=0.6$, a projection angle $\theta=10$ degrees, when varying the cosine frequency $\omega$ from 0.5 to 2.5 in increments of 0.5 , while having the amplitude fixed at $A=0.5$. As may be discerned, none of the patterns shown corresponds to a linear fractalmultifractal case. While the top pattern is reminiscent of other already found in this brief exploration, the others show another host of plausible natural sets having realistic statistical-multifractal qualifiers. Notice that, unlike the results presented in Figure 5, an increase in the parameter $\omega$ does not lead to a smoothing of an attractor. In conclusion, the sensitivity of our procedure on the parameter $\omega$ is quite complex and not intuitive, as the interplay between the iterations and the perturbation is not trivial. 
If the patterns shown were interpreted as hydrographs, observe how the second one from the top would correspond to perhaps a single event having a sustained rain. Such a pattern is also reminiscent of the width-function of natural river networks (i.e., the number of links as a function of the distance to the basin's outlet, Puente and Sivakumar, 2003).

Figure 8 illustrates how plausible width-functions may be obtained from appropriate variations in the amplitude parameters of the nonlinear perturbations of a linear (bottom) fractal-multifractal projection (Puente, 2004, Figure 6). As may be seen, the statistics of these patterns (except the power spectrum exponent) are quite similar, yet the actual distributions exhibit distinct plausible shapes that may reflect the inner structure of a natural river network. A research question inspired by these results could be to study how the parameters of the width function change as a response to erosion mechanisms. This perhaps may be addressed via variations in the parameters of the (nonlinear) projections.

As the choice of the bounded nonlinear perturbation is arbitrary, Figure 9 shows an example of what can be obtained by varying the functional shape of the nonlinear perturbation. In this example, a sigmoid function $g(y)=$ $A \cdot(1+\exp (-\omega \cdot y))^{-1}$ is used. As done before, a sensitivity analysis is presented based on the linear case used in Figure 1, varying amplitude $A$ from 0 (i.e., the linear case) to 0.5 in increments of 0.125 , while keeping the "frequency" at $\omega=2$. Notice that, for the set of parameters used, the choice of this perturbation leads to fractal attractors of increasing dimensions, which leads to increasing filtering of the parent multifractal, as reflected by an increasing correlation scale.

This graph represents just an example of the manifold possibilities of extensions that can be envisaged when looking for geometric representations of natural patterns. In this instance, as in the cosine case, further increasing the amplitude integrates the derived measure $d y$ towards a Gaussian distribution. This result is quite interesting as it generalizes the roads to Gaussianity, via space-filling fractal interpolating functions, already reported (Puente et al., 1996, 1999). Details of such a case shall reported elsewhere.

In order to further understand the nature of the results presented herein, Figure 10 shows the case when a uniformly random distribution, with varying amplitude, is used as the nonlinear perturbation. One may wonder if adding 
$A \cdot \cos (\omega \cdot y)$ to the linear maps is not equivalent to adding just a random signal, as hinted by some of the evolutions shown. However, as may be seen, increasing the amplitude of the noise tends unequivocally to locally smooth out the original signal (i.e., as in Figure 1). This means that the fractal attractor is being blurred by the addition of the noise, hence resulting in the details of the projection being lost. As just mentioned, higher values of the amplitude of the noise, for suitable values of the other parameters, lead to Gaussianity, as may naturally be expected in the context of the Central Limit Theorem (Puente, 2003).

\section{Summary}

It has been illustrated that extensions of the fractal-multifractal approach, obtained by adding nonlinear and bounded perturbations to the linear equations to be iterated, yield fractal attractors not shaped as continuous fractal interpolating functions that, nonetheless, result in a multitude of derived measures that resemble patterns found in environmental applications. Such patterns are interesting indeed in practice as they vastly extend the numerous scenarios already defined via the original framework. As reported for the linear fractal-multifractal methodology (Puente, 2004), the nonlinear extensions also provide patterns holistically encoded (at any resolution), which are defined based on only a few parameters, yielding substantial compression ratios easily exceeding 100:1.

In regards to the sensitivity cases herein, and many others not reported, the following general trends may be established: (a) the derived patterns $d y$ depend, in a non-trivial way, on the nature of the nonlinear perturbation added, whose most general form is $g(y)=A \cdot h(\omega \cdot y)$, for a suitable bounded function $h$; (b) "small" variations in the amplitude $A$ (at small constant $\omega$ ) yield more stable scenarios than "small" variations in the "frequency" $\omega ;(\mathrm{c})$ the patterns obtained by adding the nonlinear perturbations are not equivalent to those obtained by adding a white-noise perturbation, and an increase in the amplitude of the white noise progressively smooths out a given signal; and $(d)$ we have encountered many combinations of the nonlinear parameters that yield a Gaussian distribution as the derived pattern, $d y$, even though the parameters of the linear maps do not imply a space-filling attractor, as 
previously required on the linear fractal-multifractal approach; In conclusion, we have provided ample evidence that the linear and nonlinear fractal geometric procedures described in this work are a viable alternative to existing stochastic methods for the modeling of environmental information, hinting to the possibility of hidden determinism in natural complexity. The question whether simplicity may be found at the root of the complexity observed in natural systems, lies at the root of modern science: we believe that the ideas presented herein may indeed be a step in the right direction. Preliminary results regarding extensions of the nonlinear ideas to patterns over two and three dimensions also support the conclusions herein, details of which will be reported elsewhere.

It is envisioned, pending a resolution of the required inverse problem for a given observed pattern, that these notions, and other procedures aiming to capture mathematical morphology explicitly, may result in a more complete understanding of complex environmental systems and the dynamics of the patterns they produce. This is particularly so, as the evolution of records may perhaps be discerned in the compressed parameter space of subsequent sets, without the need of resorting to difficult-to-solve (stochastic) differential equations whose structure and initial conditions may not be easily determined. It is our hope that these geometry-based ideas aimed at capturing the details in the data would make further inroads in environmental research. 


\section{Acknowledgment:}

This work was partially supported by the U.S. Department of Energy under Contract No. DE-AC02-05CH11231. 


\section{References}

Barnsley MF (1986) Fractal functions and interpolation. Constr Approx 2:303-329

Barnsley MF (1988) Fractals Everywhere. Academic Press, New York

Lorenz EN (1993) The Essence of Chaos. University of Washington Press

Mandelbrot BB (1983) The Fractal Geometry of Nature. W. H. Freeman, New York

Mandelbrot BB (1989) Multifractal measures especially for the geophysicist. In: Scholz CH, Mandelbrot MM (eds). Fractals in Geophysics, Birkhauser Verlag, Basel, pp 1-42

Meneveau C, Sreenivasan KR (1987) Simple multifractal cascade model for fully developed turbulence. Phys Rev Lett 59:1424-1427

Puente CE (1992) Multinomial multifractals, fractal interpolators, and the Gaussian distribution. Phys Lett A 161:441-447

Puente CE (1994) Deterministic fractal geometry and probability. Int J Bifurc Chaos 4(6):16131629

Puente CE (1996) A new approach to hydrologic modeling: Derived distributions revisited. J Hydrol 187:65-80

Puente CE (2003) The exquisite geometric structure of a central limit theorem. Fractals $11(1): 39-52$.

Puente CE (2004) A universe projections: may Plato be Right? Chaos, Solitons Fractals $19(2): 241-253$

Puente CE, Obregón N (1996) A deterministic geometric representation of temporal rainfall. Results for a storm in Boston. Water Resour Res 32(9):2825-2839

Puente CE, Obregón N (1999) A geometric Platonic approach to multifractality and turbulence. Fractals 7(4): 403-420

Puente CE, López MM, Pinzón JE, Angulo JM (1996) The Gaussian distribution revisited. Adv Appl Prob 28(2):500-524

Puente CE, Sivakumar B (2003) A deterministic width function model. Nonlinear Processes Geophys 10:525-529

Puente CE, Sivakumar B (2007) Modeling hydrologic complexity: A case for geometric determinism. Hydrol Earth Syst Sci 11:721-724 


\section{List of Figures}

1. From a multifractal measure $d x$ to a derived measure $d y$ via a continuous fractal interpolating function from $x$ to $y$ found via the iteration of linear maps. Interpolating points: $\{(0,0),(1 / 2,-0.35),(1,-0.2)\}$, vertical scalings: $d_{1}=-0.8, d_{2}=-0.6$, parent multifractal parameter: $p=0.3$. (Measures $d x$ and $d y$ are normalized so that they add up to one).

2. From a multifractal measure $d x$ to a derived measure $d y$ via a continuous fractal interpolating function from $x$ to $y$ found via the iteration of nonlinear maps. Interpolating points: $\{(0,0),(1 / 2,-0.35),(1,-0.2)\}$, vertical scalings: $d_{1}=-0.8, d_{2}=-0.6$, parent multifractal parameter: $p=0.3$, nonlinear perturbation: $g(y)=A \cdot \cos (\omega \cdot y), A=0.5, \omega=1$.

3. Statistical and multifractal analyses of $d y$ measures corresponding to the linear (top) and nonlinear (bottom) map cases of figures 1 and 2. From left to right: projection time series made of 4096 values, autocorrelation function computed up to 1024 lags, power spectrum shown in log-log scale, and multifractal spectrum " $f$ (vertical) vs. $\alpha$ (horizontal)."

4. Sensitivity of derived measure $d y$ to variations in perturbation amplitudes $A$ for a fixed frequency $\omega$. Interpolating points: $\{(0,0),(1 / 2,-0.35)$, $(1,-0.2)\}$, vertical scalings: $d_{1}=-0.8, d_{2}=-0.6$, parent multifractal parameter: $p=0.3$, nonlinear perturbation: $g(y)=A \cdot \cos (\omega \cdot y), A=0$ (top) to $A=0.5$ (bottom) in increments of $0.125, \omega=1$. Key statistics (top to bottom): $D=1.49,1.56,1.55,1.47,1.30, D_{d y}=1.35,1.28,1.30$, $1.28,1.29, \tau_{e}=101,146,250,74,10, \beta=-1.21,-1.55,-1.45,-1.12$, $-0.98, D_{1}=0.88,0.89,0.90,0.87,0.74$.

5. Sensitivity of derived measure $d y$ to variations in perturbation frequencies $\omega$ for a fixed amplitude $A$. Interpolating points: $\{(0,0),(1 / 2,-0.35)$, $(1,-0.2)\}$, vertical scalings: $d_{1}=-0.8, d_{2}=-0.6$, parent multifractal parameter: $p=0.3$, nonlinear perturbation: $g(y)=A \cdot \cos (\omega \cdot y), A=0.5$, $\omega=0.5$ (top) to $\omega=2.5$ (bottom) in increments of 0.5 . Key statistics (top to bottom): $D=1.49,1.47,1.41,1.30,1.19, D_{d y}=1.37,1.31,1.29$, $1.29,1.31, \tau_{e}=137,80,19,10,10, \beta=-1.21,-1.08,-0.99,-0.98,-1.00$, $D_{1}=0.87,0.85,0.81,0.74,0.68$. 
6. Sensitivity of derived measure $d y$ to variations in perturbation amplitudes $A$ for a fixed frequency $\omega$. Interpolating points: $\{(0,0),(0.3,1),(1,0)\}$, vertical scalings: $d_{1}=0.4, d_{2}=-0.5$, parent multifractal parameter: $p=0.4$, projection angle: $\theta=-20$ degrees, nonlinear perturbation: $g(y)=A \cdot \cos (\omega \cdot y), A=0$ (top) to $A=1.0$ (bottom) in increments of $0.25, \omega=0.05$. Key statistics (top to bottom): $D=1.20,1.20,1.20$, $1.15,1.12, D_{d y}=1.42,1.42,1.40,1.42,1.38, \tau_{e}=14,27,25,25,25$, $\beta=-1.14,-1.08,-1.13,-1.07,-1.11, D_{1}=0.91,0.90,0.88,0.88,0.76$.

7. Sensitivity of derived measure $d y$ to variations in perturbation frequencies $\omega$ for a fixed amplitude $A$. Interpolating points: $\{(0,0),(1 / 2,5),(1,4)\}$, vertical scalings: $d_{1}=0.9, d_{2}=-0.4$, parent multifractal parameter: $p=0.6$, projection angle: $\theta=10$ degrees, nonlinear perturbation: $g(y)=$ $A \cdot \cos (\omega \cdot y), A=0.5, \omega=0.5$ (top) to $\omega=2.5$ (bottom) in increments of 0.5. Key statistics (top to bottom): $D=1.19,1.50,1.53,1.13,1.54$, $D_{d y}=1.37,1.44,1.41,1.29,1.30, \tau_{e}=85,417,448,262,120, \beta=-1.11$, $-1.00,-1.58,-1.27,-1.00, D_{1}=0.81,0.94,0.91,0.86,0.85$.

8. Sensitivity of derived measure $d y$ to variations in perturbation amplitudes $A$ for a fixed frequency $\omega$. Interpolating points: $\{(0,0),(1 / 2,1),(1,0)\}$, vertical scalings: $d_{1}=0.7, d_{2}=-0.7$, parent multifractal parameter: $p=0.5$, projection angle: $\theta=20$ degrees, nonlinear perturbation: $g(y)=$ $A \cdot \cos (\omega \cdot y), A=-0.5$ (top) to $A=0$ (bottom) in increments of 0.125 , $\omega=0.5$. Key statistics (top to bottom): $D=1.57,1.56,1.55,1.55$, $1.55, D_{d y}=1.49,1.49,1.49,1.49,1.50, \tau_{e}=222,210,216,218,224$, $\beta=-0.66,-0.66,-0.72,-0.75,-0.80, D_{1}=0.99,0.99,0.99,0.99,0.99$.

9. Sensitivity of derived measure $d y$ to variations in perturbation amplitudes $A$ for a fixed frequency $\omega$. Interpolating points: $\{(0,0),(1 / 2,-0.35)$, $(1,-0.2)\}$, vertical scalings: $d_{1}=-0.8, d_{2}=-0.6$, parent multifractal parameter: $p=0.3$, projection angle: $\theta=0$ degrees, nonlinear perturbation: $g(y)=A \cdot\left(1+e^{-\omega \cdot y}\right)^{-1}, A=0$ (top) to $A=0.5$ (bottom) in increments of $0.125, \omega=2$. Key statistics (top to bottom): $D=1.50$, $1.57,1.62,1.67,1.72, D_{d y}=1.35,1.30,1.27,1.22,1.26, \tau_{e}=101,218$, $228,353,394, \beta=-1.21,-1.51,-1.57,-1.66,-1.43, D_{1}=0.88,0.91,0.88$, $0.89,0.89$. 
10. Sensitivity of derived measure $d y$ to variations in perturbation amplitudes $A$ of a uniform noise perturbation. Interpolating points: $\{(0,0),(1 / 2,-$ $0.35),(1,-0.2)\}$, vertical scalings: $d_{1}=-0.8, d_{2}=-0.6$, parent multifractal parameter: $p=0.3$, projection angle: $\theta=0$ degrees, noise characteristics: $A=0$ (top) to $A=0.005$ (bottom) in increments of 0.00125 . Key statistics (top to bottom): $D=1.50,1.50,1.51,1.52,1.53, D_{d y}=1.35$, $1.38,1.40,1.39,1.40, \tau_{e}=101,174,192,219,234, \beta=-1.21,-1.60$, $-1.15,-0.97,-0.82, D_{1}=0.88,0.90,0.91,0.92,0.92$. 

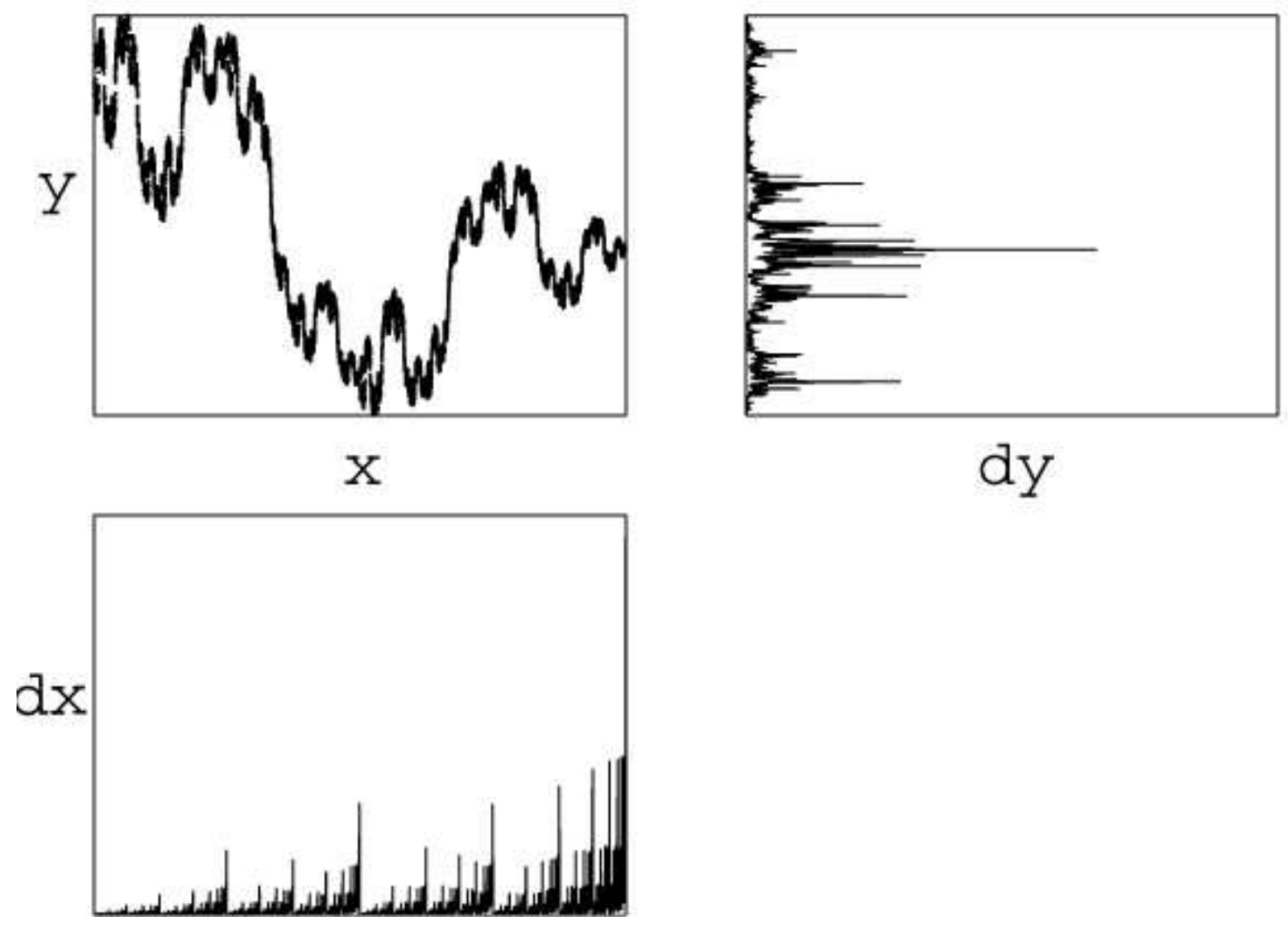

Figure 1 

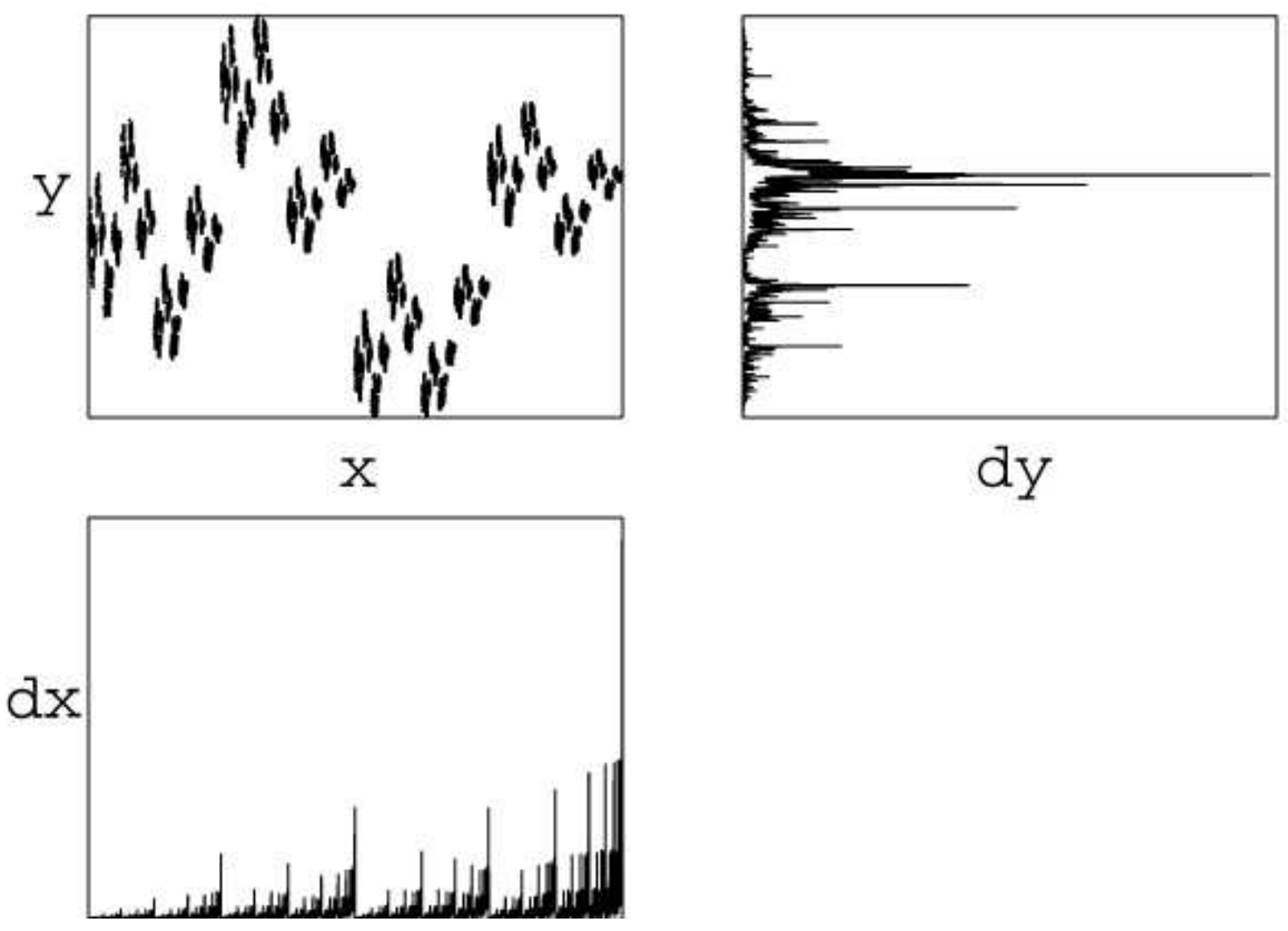

Figure 2 

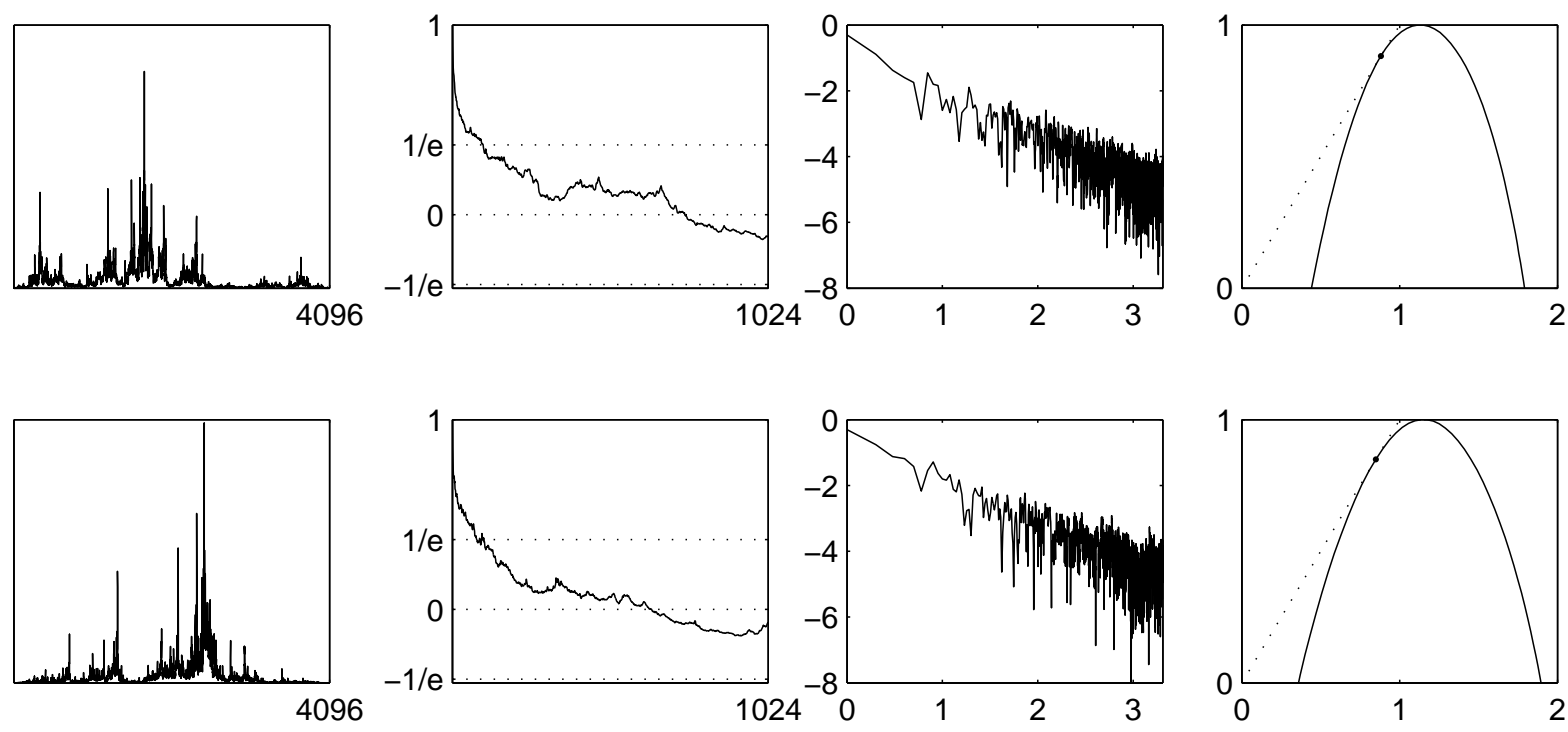

Figure 3 

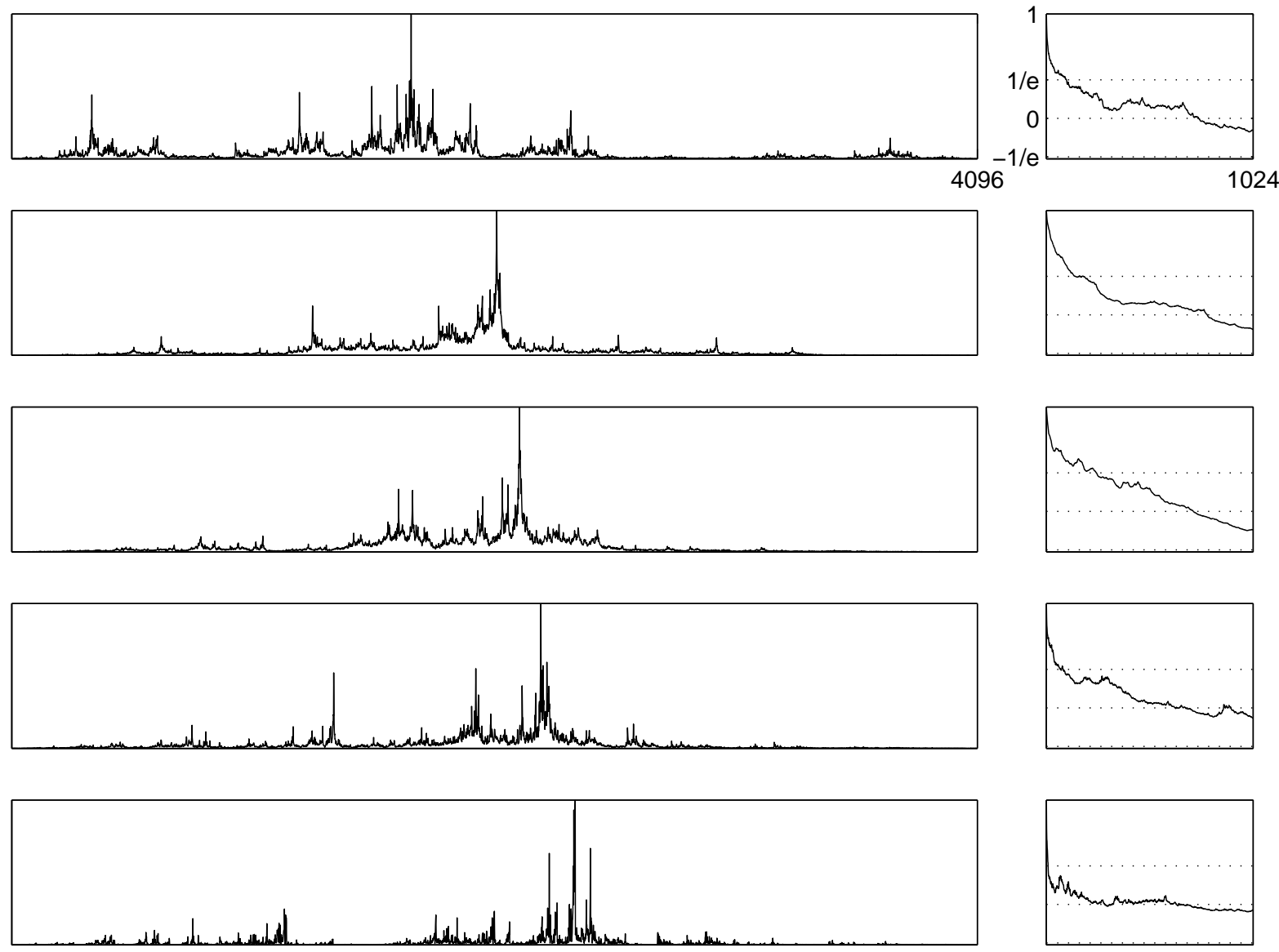

Figure 4 

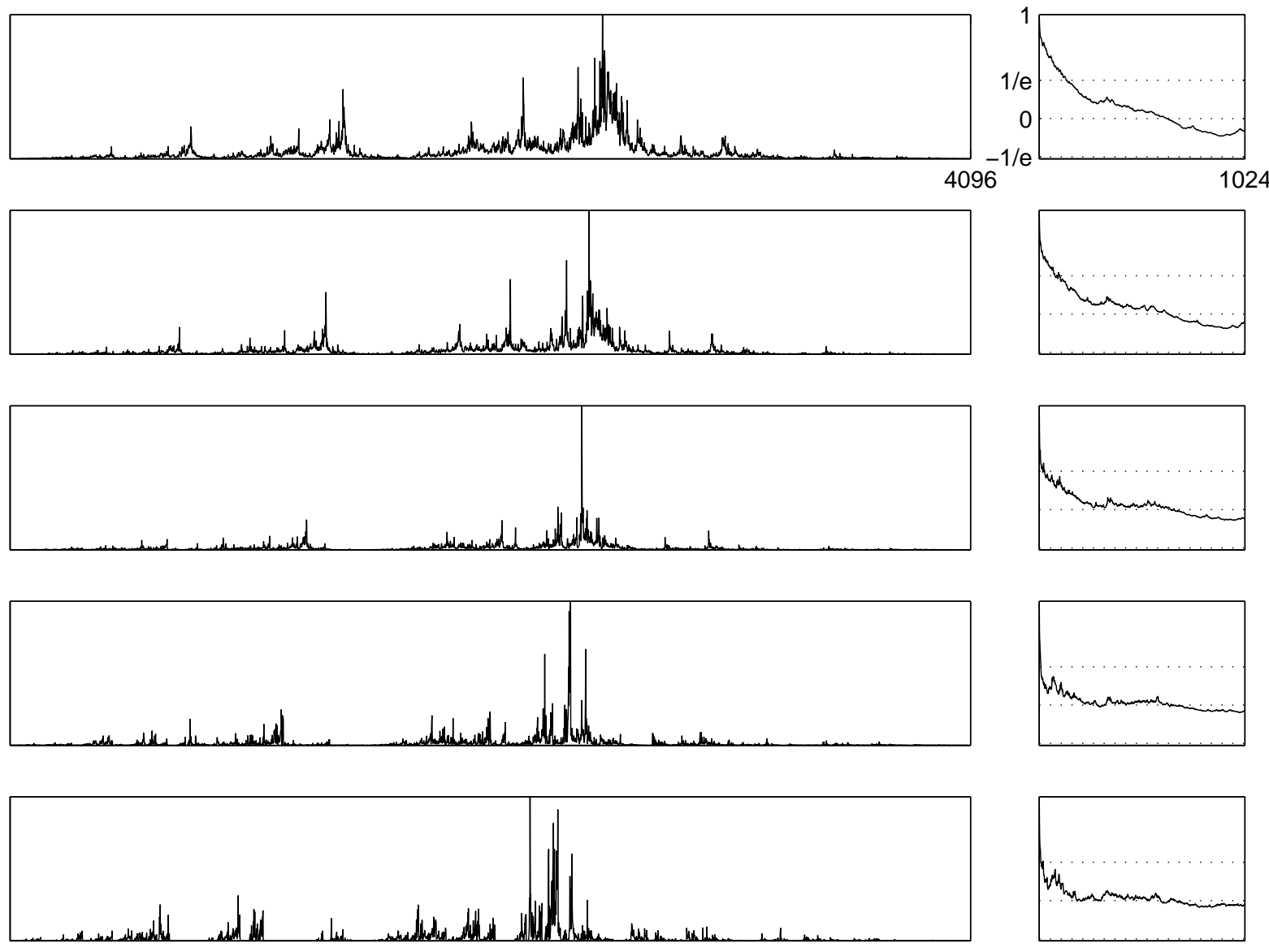

Figure 5 

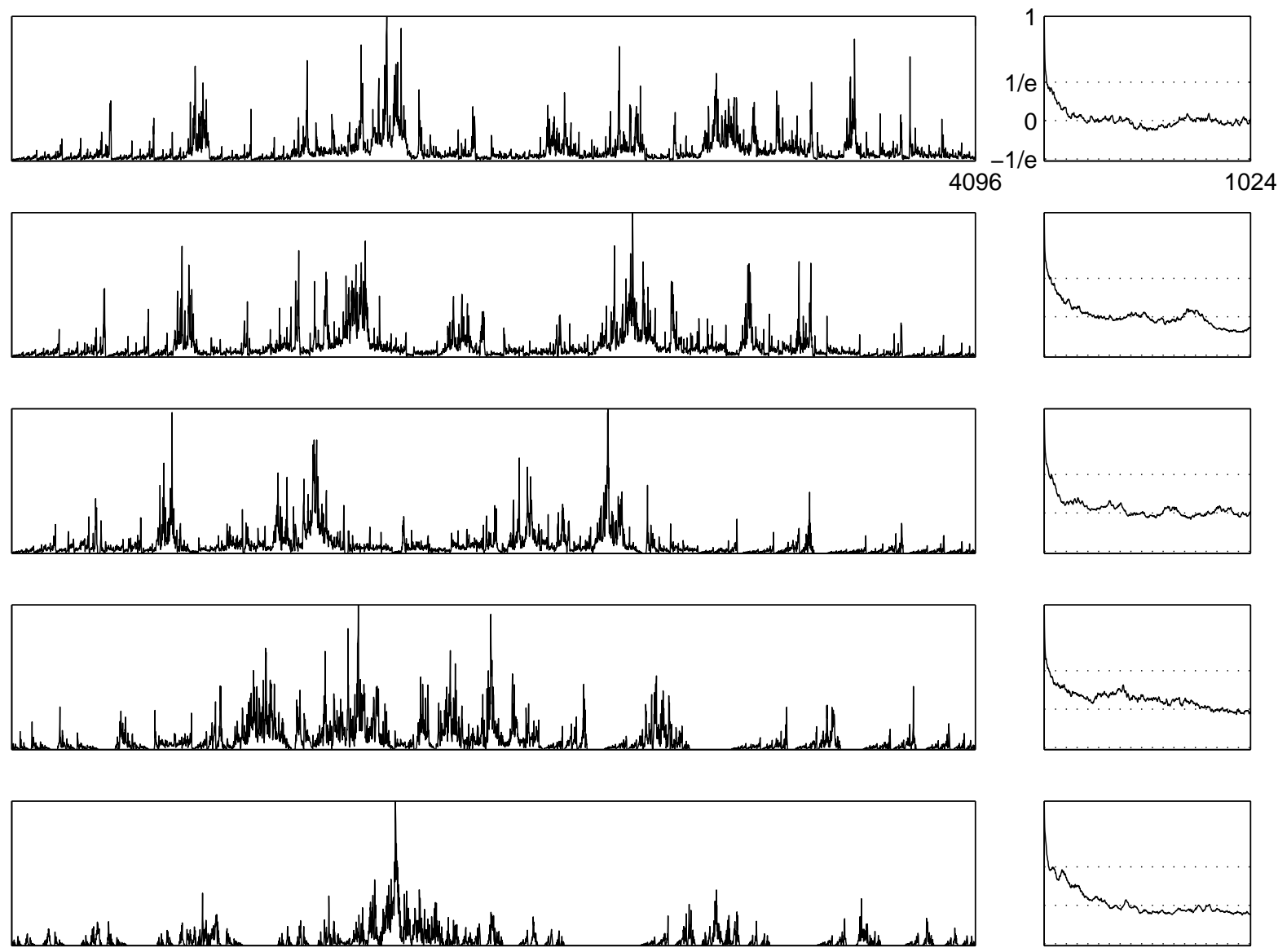

Figure 6 

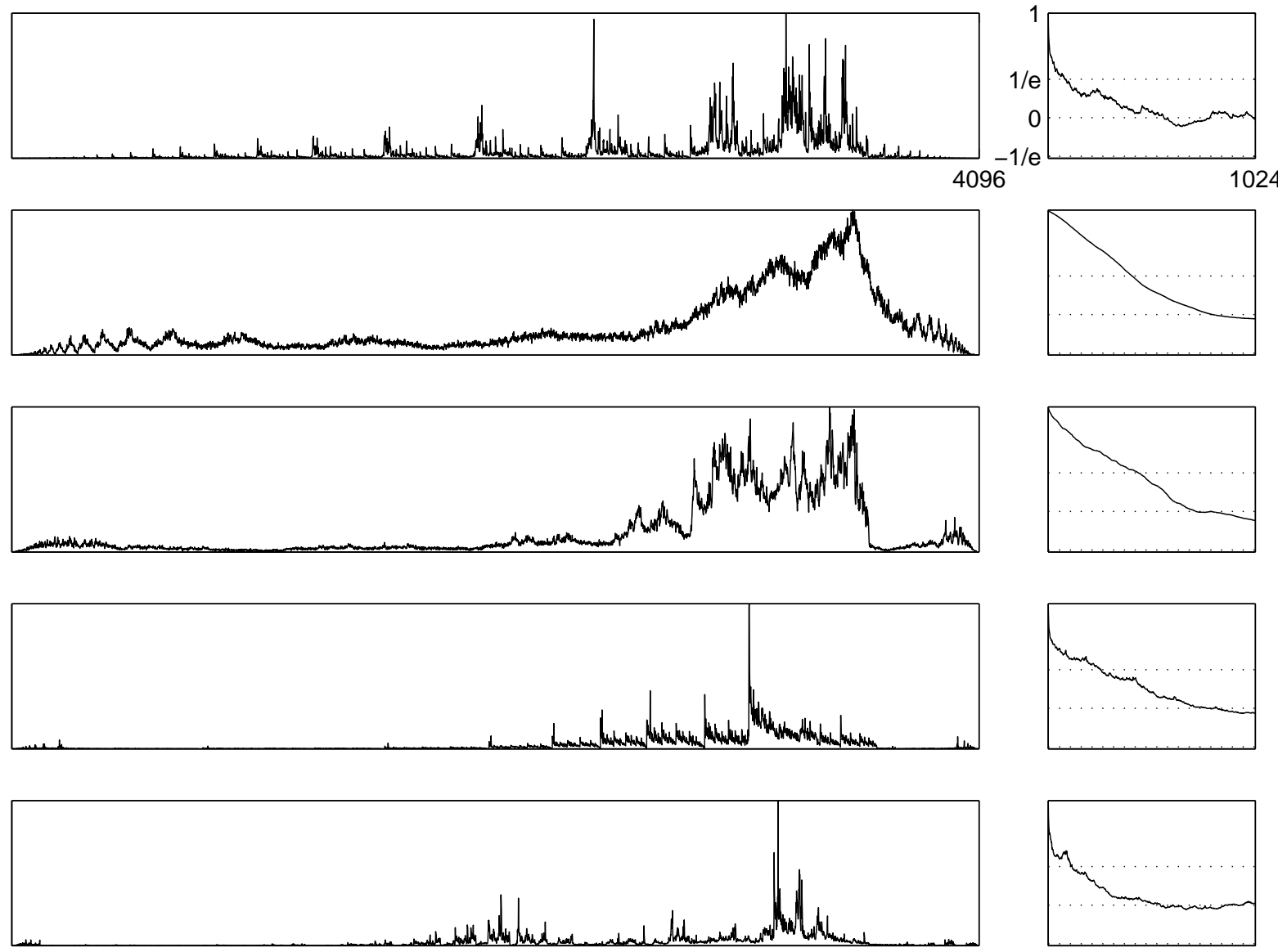

Figure 7 

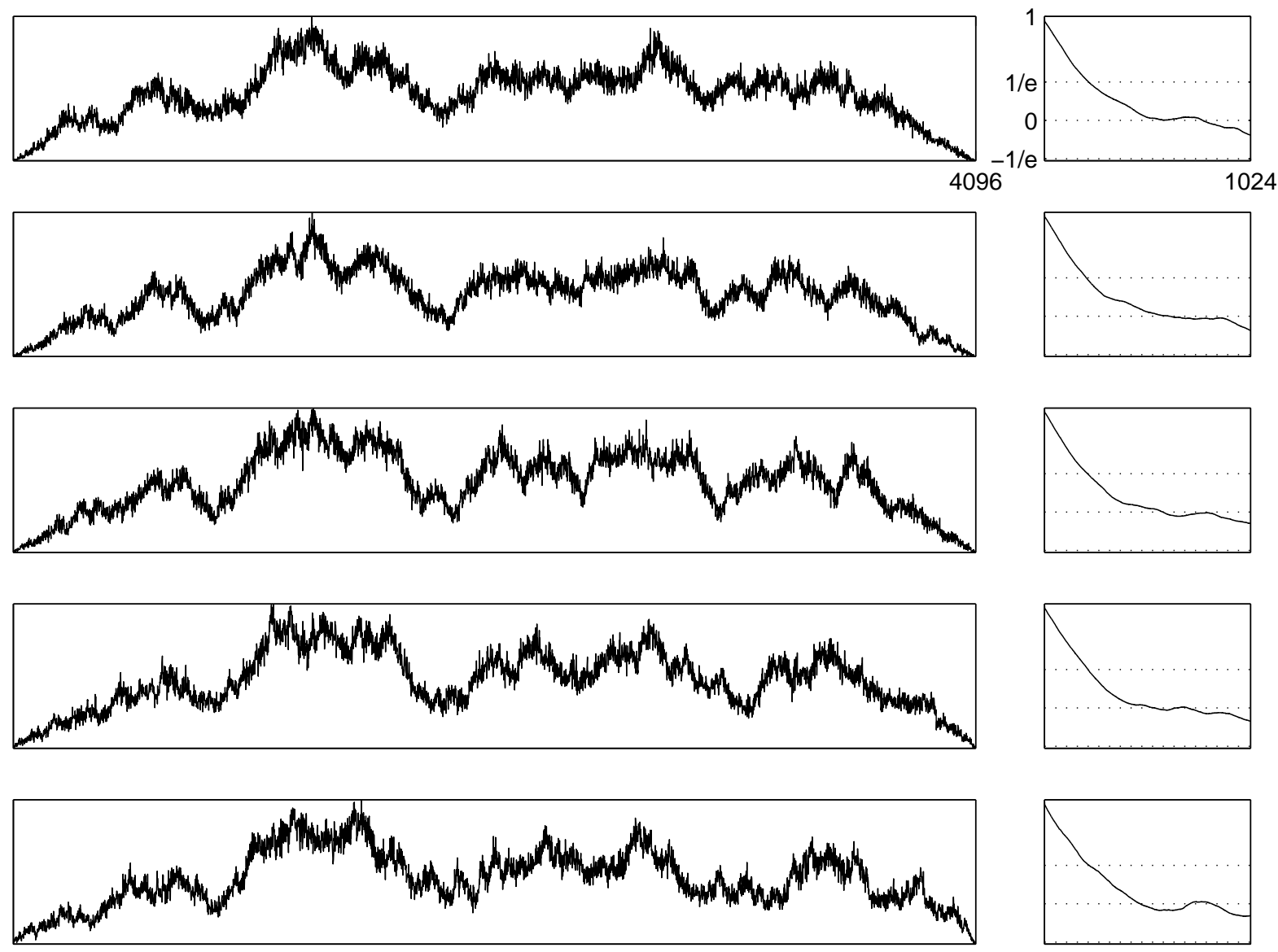

Figure 8 

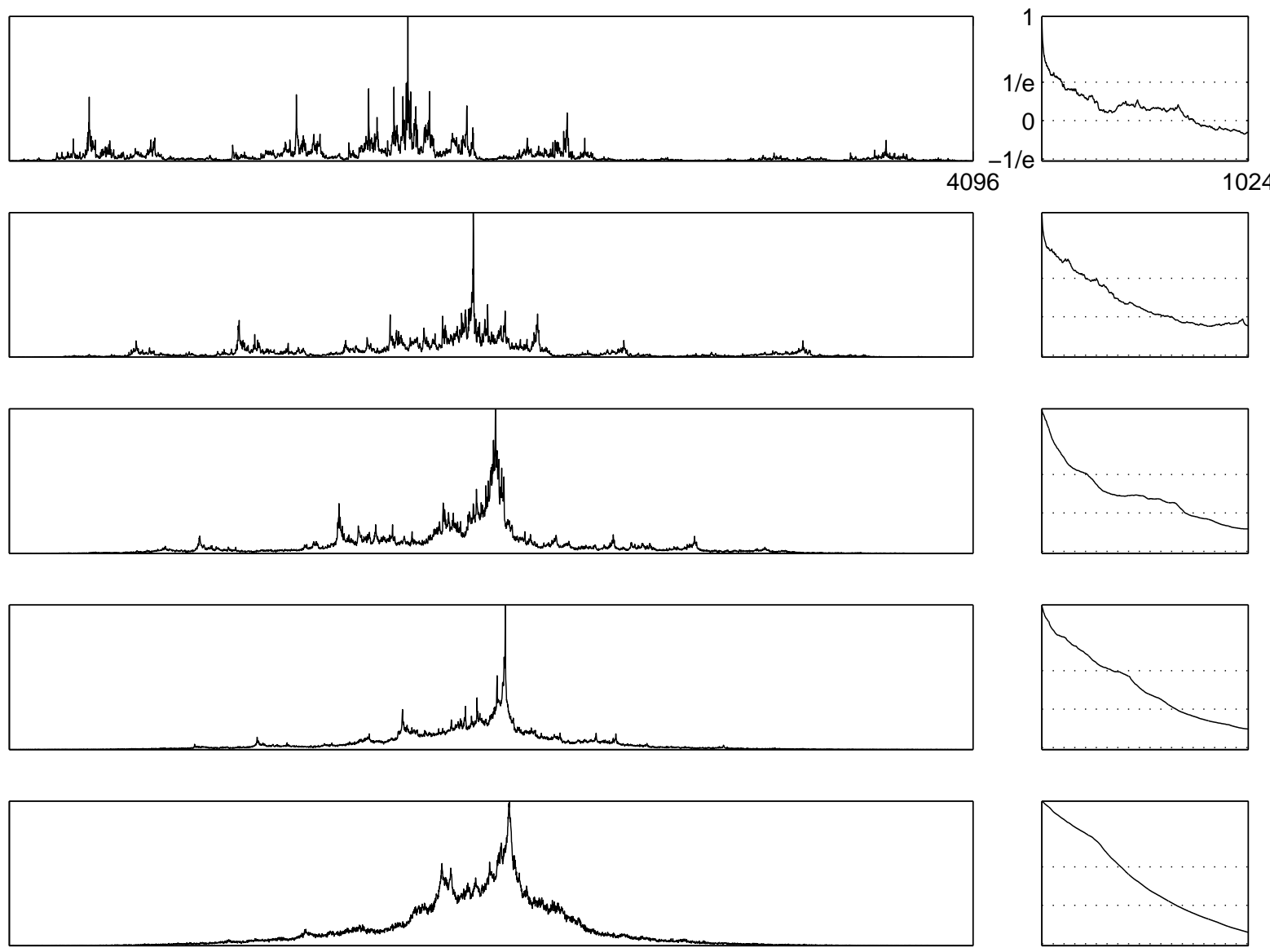

Figure 9 

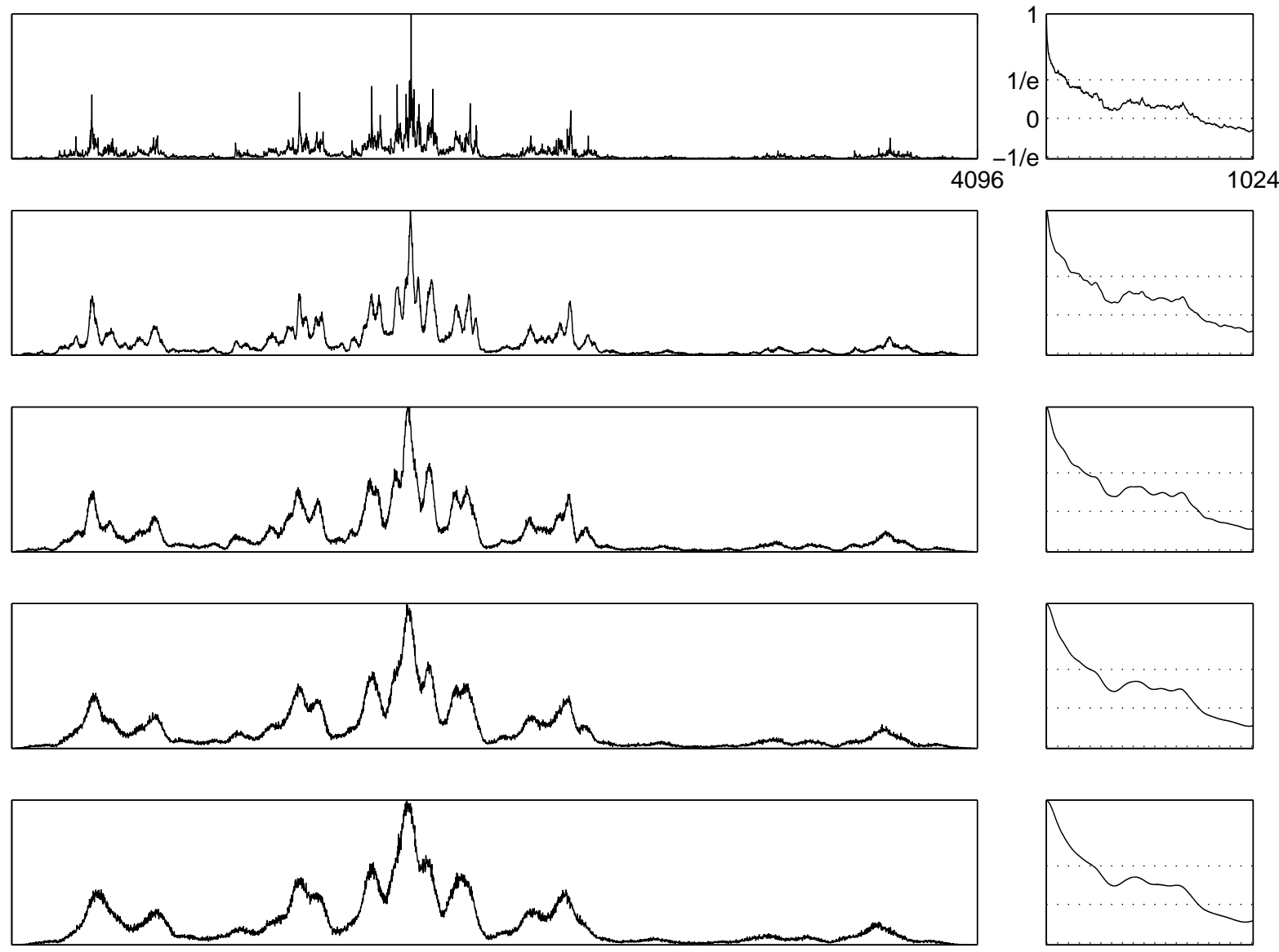

Figure 10 